\title{
SIMULAÇÃO DA CINÉTICA DE REAÇÃO SUPERFICIAL DA EXTRAÇÃO DO ÍON COBRE UTILIZANDO CONTACTORES POR MEMBRANAS
}

\author{
R. T. BAIA ${ }^{1}$, J. A. da SILVA ${ }^{1}$ e K. B. OLIVEIRA ${ }^{2}$ \\ ${ }^{1}$ Universidade do Estado do Amapá, Departamento de Engenharia Química \\ 2 Universidade Federal do Pará, Faculdade de Engenharia Química \\ E-mail para contato: renanbaiatei@gmail.com
}

\begin{abstract}
RESUMO - A técnica de separação por membranas vem ganhando destaque nas pesquisas e em algumas indústrias por apresentarem eficiência, seletividade e economia de energia, dentre outras qualidades. A extração de metais nobres através do uso de membranas de fibra oca é uma das técnicas bem sucedidas, pois possibilita a extração do metal de interesse com menor quantidade de solvente e economia de capital, além de poder ser usada para reaproveitar partículas do metal nobre presente nos resíduos da mineração, gerando um maior rendimento e menor impacto ambiental. O presente trabalho tem por objetivo estudar e simular a cinética de extração do íon cobre através do processo de separação por membranas de fibra-oca e calcular a eficiência dessa extração. O software utilizado para realizar as simulações foi o COMSOL Multiphysics ${ }^{\circledR}$ v. 4.4. O modelo cinético que melhor se ajustou aos dados experimentais foi o para reação superficial de segunda ordem irreversível, de acordo com a literatura. O erro estimado médio calculado foi $6,399 \%$. O valor máximo para a eficiência da extração foi $95,18 \%$.
\end{abstract}

\section{INTRODUÇÃO}

Membranas e processos com membranas podem efetuar a separação de certas misturas moleculares efetivamente e economicamente em temperatura ambiente, sem nenhum efeito tóxico ou reações prejudiciais para os produtos (Pabbly et al., 2009). Além de oferecerem economia de energia, seletividade, podem trabalhar com substâncias termossensíveis - como fármacos e produtos alimentícios -, também são de fácil operação e precisam de pouca mão de obra (Habert et al., 2006; Kislik, 2010).

Entre os vários tipos de membrana, destacam-se os contactores por membranas de fibra oca por possuírem elevada área superficial em relação ao volume que ocupam, instalações compactas e pequenas. Nessas membranas, a área interfacial de transferência de massa é muito maior e melhor controlada, a natureza hidrofílica e hidrofóbica da membrana determina a posição da interface entre a alimentação e o solvente (Kislik, 2010; Shirazian et al., 2011).

Há vários anos a técnica de separação por membranas vem ganhando destaque nas pesquisas e em algumas indústrias, vários autores vem tratando do tema de extração de metais nobres através do uso de membranas de fibra oca e alcançando bons resultados, como menor 


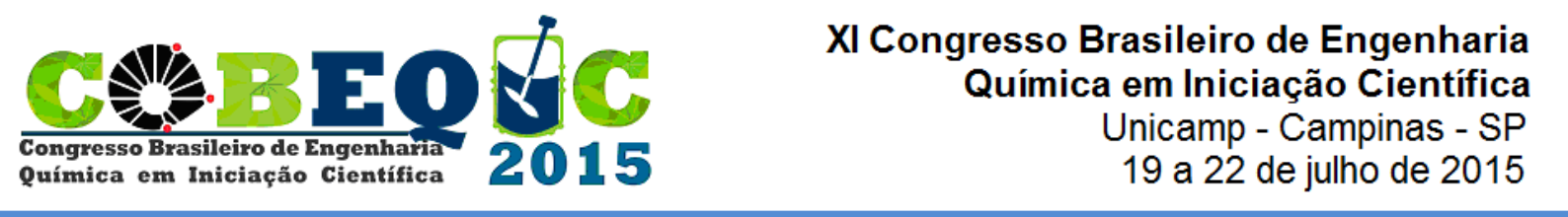

quantidade de solvente, boa eficiência e economia de capital (Fadaei et al., 2011, Gharidi e Shirazian, 2013).

O objetivo deste estudo é estudar a simulação da cinética de reação superficial da extração do íon cobre utilizando contactores por membranas, determinar as constantes de reação e calcular a eficiência do processo de extração.

\section{REFERENCIAL TEÓRICO}

\subsection{Estrutura da Membrana}

A performance da membrana está estreitamente relacionada com a sua estrutura, material e módulo (Lothongkum et al., 2011).

No caso do material, as membranas podem ser feitas de vários polímeros (por exemplo, acetato de celulose, poliamida, polipropileno), são baratas, fáceis de fabricar e possuem vasta gama de tamanhos de poros. No entanto, existem algumas limitações, como $\mathrm{pH}$, temperatura e pressão, que podem impedir a utilização de membrana polimérica (Nataya e Paisan, 2014).

O módulo é o elemento básico de um sistema de membrana. É nele que estão todas as estruturas necessárias para viabilizar a separação de um Processo de Separação por Membranas (Oliveira, 2013).

O módulo de fibra-oca é formado por várias membranas cilíndricas, longas e finas, presas nas extremidades por placas, inseridas em um tubo maior. A alimentação entra por uma extremidade e percorre o interior dos tubos e é retirada na outra extremidade após ser permeada. As principais vantagens desta configuração são: alta superfície de contato por unidade de volume, facilidade de operação e manutenção, e o baixo consumo de energia (Mulder, 1996).

\subsection{Mecanismo de Transporte da Extração do íon Cobre}

O módulo é alimentado por duas soluções que escoam em contracorrente, uma é a fase de alimentação (solução problema) que contém os íons de cobre $\left(\mathrm{Cu}^{2+}\right)$, e a outra é constituída pelo extratante orgânico LIX84-I (HR) dissolvido em querosene (Pancharoen et al., 2011)

A reação de extração ocorre na interface dos poros da membrana de fibra-oca quando as duas fases entram em contato, conforme o esquema da Figura 1, dando origem ao complexo $\left(\mathrm{CuR}_{2}\right)$ e liberando íon hidrogênio $\left(\mathrm{H}^{+}\right)$, de acordo com a Equação 1. O complexo é transportado por difusão através da membrana e depois por convecção na fase orgânica, enquanto que o $\mathrm{H}^{+}$é transportado pela fase aquosa (Kislik, 2010, Nattaya, 2014).

$$
\mathrm{Cu}^{2+}+2 \mathrm{HR} \rightleftarrows \mathrm{CuR} \mathrm{R}_{2}+2 \mathrm{H}^{+}
$$


Figura 1 - Esquema representativo do módulo de extração contendo uma fibra-oca (Oliveira, 2013).

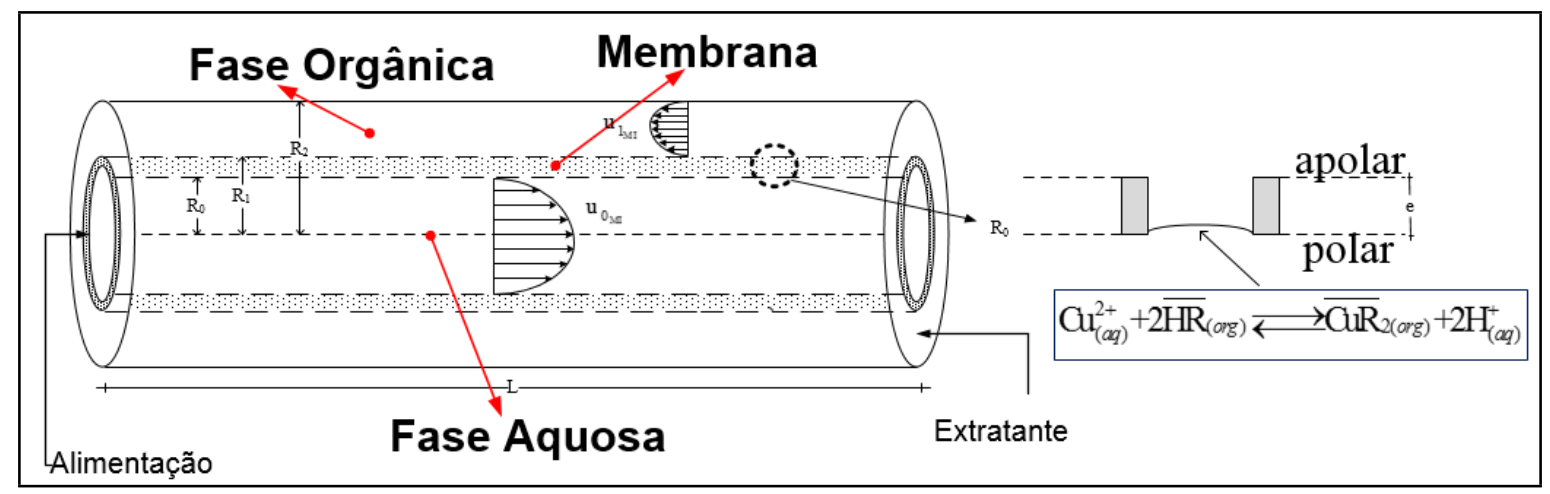

\section{METODOLOGIA}

As simulações foram realizadas no software COMSOL Multiphysics ${ }^{\circledR}$ v4.4 através da ferramenta Engenharia das Reações, considerando-se o processo de extração em regime transiente.

Abordou-se o problema com três metodologias distintas, cada uma considerando um tipo de cinética de reação e calculou-se o erro médio relativo $(\mathrm{E} \%)$ e o coeficiente de determinação $\left(\mathrm{R}^{2}\right)$ de cada uma para se analisar qual cinética oferece os dados teóricos mais aproximados aos dados experimentais. Essas cinéticas foram: reação de superfície irreversível de primeira ordem, reação de superfície irreversível de segunda ordem e reação de superfície reversível elementar de segunda ordem. Foram calculados os valores das constantes cinéticas para cada reação.

Para as reações de superfície irreversíveis foi utilizada a Equação 2 e para sua taxa de formação foi usada a Equação 3, sendo que $n$ representa a ordem da reação, $k_{l}$ é a constante cinética e $r a$ é a taxa de reação.

$$
\begin{aligned}
& A+2 B \stackrel{k 1}{\longrightarrow} C+2 D \\
& -r_{A}=k_{1} C_{A}^{n}
\end{aligned}
$$

Para a reação de superfície reversível elementar de segunda ordem foi utilizada a Equação 4 e para sua taxa de formação foi usada a Equação 5.

$$
\begin{aligned}
& A+2 B \underset{k-1}{\stackrel{k 1}{\rightleftarrows}} C+2 D \\
& -r_{A}=k_{1} C_{A} C_{B}^{2}-k_{-1} C_{C} C_{D}^{2}
\end{aligned}
$$

Onde A é o íon Cobre, B é LIX84I, C é o complexo, D é o íon hidrogênio, $r_{A}$ é a taxa de formação do metal na superfície onde ocorre a reação, k1 e k-1 são as constantes cinéticas de reação na ordem direta e inversa, respectivamente. 
O grau de ajuste de cada modelo considerou a magnitude do coeficiente de determinação $\left(\mathrm{R}^{2}\right)$, os percentuais de extração de íons de cobre a partir de dados experimentais obtidos na literatura e do modelo foram calculados pela Equação 6. O erro médio relativo foi calculado pela Equação 7.

$$
\begin{aligned}
& \% \text { Extração }=\frac{C_{\mathrm{A}, \text { entra }}-C_{\mathrm{A}, \text { sai }}}{C_{\mathrm{A}, \text { entra }}} \\
& E \%=\frac{100}{\mathrm{~N}} \sum \frac{\left|\mathrm{Y}-\mathrm{Y}^{\prime}\right|}{\mathrm{Y}}
\end{aligned}
$$

Na qual, Y é o valor observado experimentalmente, $Y^{\prime}$ é o valor calculado pelo modelo, $\mathrm{N}$ e o número de parâmetros do modelo.

\section{RESULTADOS E DISCUSSÕES}

As propriedades e condições experimentais de operação são: a membrana é constituída de polipropileno, com porosidade de $30 \%$ e tortuosidade de $2,6 \mathrm{~T}$, o número de fibras é aproximadamente de 2200, a densidade de empacotamento é $7976\left(\mathrm{~m}^{2} / \mathrm{m}^{3}\right)$, a concentração inicial de cobre é $1,194 \mathrm{~g} / \mathrm{L}$ e vazão a de alimentação de $1,94 \mathrm{~cm}^{3} / \mathrm{s}$, com uma temperatura de $25^{\circ} \mathrm{C}$ e tempo de trabalho de 3600 segundo.

A partir dos resultados das simulações foram obtidos os valores das constantes cinética, os erros médios relativos $(\mathrm{E} \%)$ e os coeficientes de determinação $\left(\mathrm{R}^{2}\right)$ apresentados na Tabela 1.

Tabela 1 - Valores de $\mathrm{R}^{2}$, erros médios relativos (E\%) e as constantes cinéticas para os modelos de reações superficiais de primeira ordem, segunda ordem reversível e irreversível, respectivamente.

\begin{tabular}{c|c|c|c|c}
\hline Modelo & $\mathbf{k}_{\mathbf{1}}$ & $\mathbf{k}_{\mathbf{- 1}}$ & $\mathbf{E \%}$ & $\mathbf{R}^{\mathbf{2}}$ \\
\hline $\begin{array}{c}\text { Primeira ordem } \\
\text { irreversível }\end{array}$ & $2,97 \mathrm{E}-03 \mathrm{~m} / \mathrm{s}$ & - & 24,4429 & 0,9495 \\
\hline $\begin{array}{c}\text { Segunda ordem } \\
\text { Irreversível }\end{array}$ & $2,92 \mathrm{E}-04 \mathrm{~m}^{4} / \mathrm{mol}^{*} \mathrm{~s}$ & - & 6,3990 & 0,9679 \\
\hline $\begin{array}{c}\text { Segunda ordem } \\
\text { Reversível }\end{array}$ & $1,446 \mathrm{E}-06 \mathrm{~mol}^{7} / \mathrm{mol}^{*} \mathrm{~s}$ & $5,9858 \mathrm{E}-8 \mathrm{~mol}^{7} / \mathrm{mol}^{*} \mathrm{~s}$ & 9,5511 & 0,9677 \\
\hline
\end{tabular}

Percebe-se que, os valores da Tabela 1 sugerem que o modelo que mais se adequou aos dados experimentais foi da reação superficial de segunda ordem irreversível, estando de acordo com as literaturas, como os trabalhos de Pancharoen et al. (2011) e Oliveira (2013). Os valores do erro médio e $\mathrm{R}^{2}$ da simulação foram respectivamente, 6,3990\% e 0,9679.

A Figura 2.a mostra a comparação do modelo com os dados experimentais das concentrações do cobre e do complexo em função do tempo em segundos, observa-se que os pontos experimentais ficaram bem próximos ao modelo predito pelo COMSOL. A Figura 2.b mostra a taxa de formação do íon cobre, os pontos estão próximo à linha e o valor do $\mathrm{R}^{2}$ foi de 
0,967891, indicando que os valores experimentais estão em conformidade com o modelo. Ambas as figuras citadas acima foram obtidas da cinética de reação superficial de segunda ordem irreversível.

Figura 2 - a) concentrações do cobre e do complexo em função do tempo, b) gráfico com as taxa de formação do íon cobre.
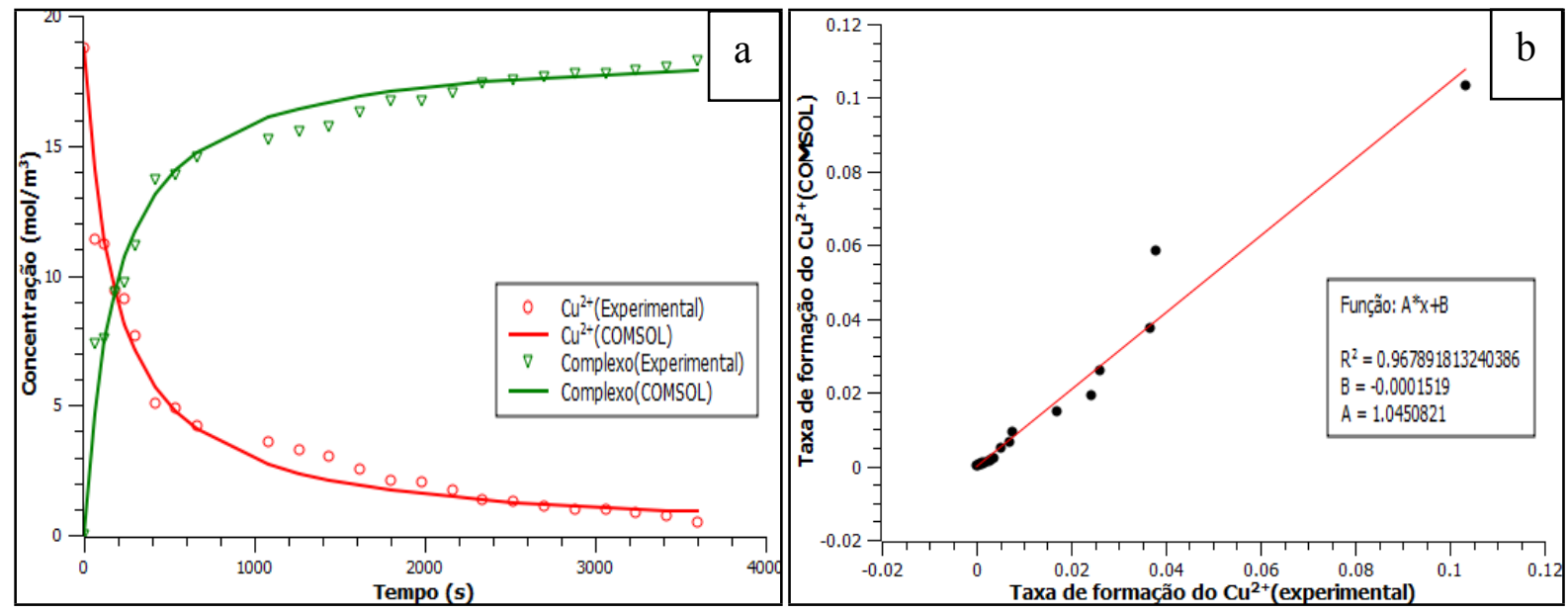

O erro médio relativo da eficiência extração do íon cobre pelo modelo do COMSOL com os dados do experimento foi de 4,0735\%, sendo que os valores máximos da eficiência da extração do experimento e simulado foram bem aproximados com 97,2194\% e 95,1869\%, respectivamente. A Figura 3 mostra a eficiência da extração do íon cobre extração.

Figura 3 - Eficiência da extração do íon cobre

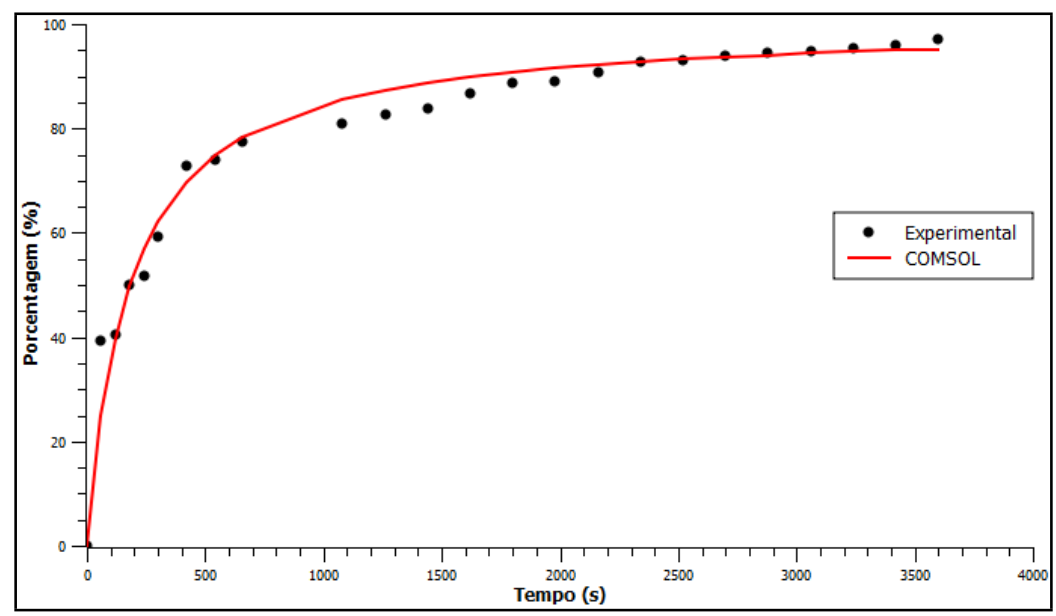




\section{CONCLUSÃO}

O COMSOL Multiphysics v.4.4 mostrou ser uma ferramenta muito útil e além de ser eficaz para simular problemas de cinética de reação superficial no processo de separação por membranas utilizando contactores por membranas através de módulo de membranas poliméricas do tipo fibras ocas. Os resultados obtidos nas simulações foram bastante satisfatórios, validados com dados experimentais e de acordo com a literatura, proporcionando assim uma boa predição da concentração do cobre na cinética de segunda ordem irreversível tendo um baixo erro relativo médio.

\section{REFERÊNCIAS}

FADAEI, F; ASHRAFIZADEH, S. N.; SHIRAZIAN, S. Mass transfer simulation of solvent extraction in hollow-fiber membrane contactors. Desalination v. 275, p. 126-132, 2011.

GHARIDI, M.; SHIRAZIAN, S. Computational simulation of mass transfer in extraction of alkali metals by means of nanoporous membrane extractors. Chemical engineering and processing, v.69, p. 57-62, 2013.

HABERT, A. C.; BORGES, C. P.; NOBREGA, R. Processos de separação por membranas. Rio de Janeiro: Editora Epapers, 2006.

KISLIK, V. S. Liquid Membranes: Principles \& Applications in Chemical. 5nd edition. Elsevier, 2010.

LOTHONGKUM, A.W; PANCHAROEN, U.; PRAPASAWAT, T. "Roles of facilitated transport through HFSLM in engineering applications," p.177-180, 2011.

MULDER, J. Basic Principles of Membrane Technology. Ed. Springer Netherlands, 1996

NATTAYA, L.; PAISAN, K. Modeling and simulation of palladium-ion extraction via hollow fiber supported liquid membrane, International Journal of Technical Research and Applications v.2, p. 37-45, 2014.

OLIVEIRA, K. O. Estudo da extração de cobre utilizando contactores por membranas como alternativa ao processo convencional de extração por solvente. Tese (Doutorado)Universidade Federal do Pará. Instituto de Tecnologia. Programa de Pós-Graduação em Engenharia de Recursos Naturais da Amazônia, 2013.

PANCHAROEN, U.; WONGSAWA, T.; LOTHONGKUM A. W. A reaction flux model for extraction of $\mathrm{Cu}(\mathrm{II})$ with LIX84I in HFSLM. Separation Science and Technology, v.46, p. 2183-2190, 2011.

SHIRAZIAN, S.; MARJANI, A.; FADAEI, F. Supercritical extraction of organic solutes from aqueous solutions by means of membrane contactors: CFD simulation. Desalination, v.277, p. 135-140, 2011. 